\title{
Characteristics of R-R intervals in patients with continuous atrial fibrillation and subsequent heart failure
}

\author{
Naoto Yoneda ${ }^{1}$, Hiroshi Takaishi ${ }^{1}$, Daisuke Matsumoto ${ }^{1}$, Toshiya Kataoka ${ }^{1}$, Yasushi \\ Tanaka $^{1}$, Makito Ozawa ${ }^{1}$, Makiko Suto $^{1}$, and Nao Shibata ${ }^{1}$ \\ ${ }^{1}$ Yodogawa Christian Hospital
}

May 11, 2020

\begin{abstract}
Introduction: Atrial fibrillation (AF) predisposes patients to heart failure (HF) and a resultant increase in mortality, however, little is known regarding what characteristics of AF are related to HF. Our aim is to clarify the characteristics of AF with subsequent HF by analysis of R-R interval during every 1-h segment. Method and Results: We retrospectively analyzed 188 Holter electrocardiograms of patients with continuous AF. A histogram of normal R-R intervals was plotted for each hour, the mean R-R intervals (mean R-R) and the standard deviation of $\mathrm{R}-\mathrm{R}$ intervals (SD R-R) were calculated. Mean R-R and SD R-R demonstrated circadian variations in 167 patients without subsequent HF. In 21 patients with subsequent HF, SD R-Rs were significantly shorter than those in patients without $\mathrm{HF}$ at 1:00-2:00 (174.9 \pm 46.4 vs. $207.3 \pm 67.5 \mathrm{~ms})$, at 2:00-3:00 $(180.4 \pm 42.8$ vs. $212.7 \pm 68.1 \mathrm{~ms})$, at 3:00-4:00 (181.9 \pm 52.7 vs. $216.1 \pm 71.0 \mathrm{~ms})$, and at 4:00-5:00 (187.9 \pm 50.0 vs. 220.7 $\pm 72.0 \mathrm{~ms}$ ). Mean R-R and SD R-R kept in low values during night-time in $\beta$-blocker users of the HF group. The attenuation of circadian variations of mean R-R and SD R-R were also recognized in patients with reduced ejection fraction. Conclusions: The analyses of $\mathrm{R}-\mathrm{R}$ interval under the consideration of the circadian variations and the usage of $\beta$-blocker were suspected to predict subsequent HF in patients with continuous AF.
\end{abstract}

\section{Hosted file}

main document-2.docx available at https://authorea.com/users/320607/articles/450146characteristics-of-r-r-intervals-in-patients-with-continuous-atrial-fibrillation-andsubsequent-heart-failure 